\title{
The further development of musculoskeletal ultrasonography for sports injuries
}

\author{
Hideyuki Goto ${ }^{1}$
}

Received: 24 April 2021 / Accepted: 30 April 2021

(c) The Japan Society of Ultrasonics in Medicine 2021

In recent years, use of musculoskeletal ultrasonography (MSKUS) has become widespread, and among them, MSKUS for sports injuries has become popular. In the case of sports injuries, the frequency of injuries to soft tissues such as muscles, ligaments, and tendons is relatively high, and magnetic resonance imaging (MRI) and ultrasonography (US) are useful for their evaluation. With MRI, however, it is difficult to perform frequent examinations due to the waiting time before the examination, the imaging time, and the high cost, and in some cases the examination cannot be performed due to claustrophobia or the presence of metal materials in the body.

On the other hand, US has many advantages. With advances in devices, ultrasonic images have become clearer, and the accuracy has surpassed that of MRI. The devices have become more compact and can be used not only in outpatient clinics but also at sporting event sites. There is also a way to share information on the pathological condition by facing the athlete and explaining while scanning the injured part. In the treatment of chronic tendon attachment disorders, which are common in sports injuries, high-quality treatment can be performed by injection into an appropriate site under ultrasound guidance. It is cheaper than other diagnostic imaging equipment and can be used multiple times.

There is a growing need for young orthopedic surgeons and medical staff to utilize US examinations, which have many advantages, in sports practice. However, I have to say that the education system is still inadequate. In the field of ultrasonic medicine, examination techniques have been developed for each clinical department and each organ. However, the parts evaluated by MSKUS are diverse throughout the body, and there are many imaging methods and conditions according to the characteristics of individual

Hideyuki Goto

hgoto@sgk.ac.jp

1 Department of Sports and Fitness, Faculty of Wellness, Shigakkan University, Aichi, Japan components such as muscles, joints, ligaments, and neurovascular bundles in each part. Therefore, to improve one's MSKUS skills, it is necessary to learn the appropriate imaging method according to each part. In addition, ultrasound-guided injections and surgical procedures are being performed as minimally invasive treatments for athletes. Under such circumstances, it is considered essential to create an educational system for efficiently acquiring skills. In particular, the target tissue for MSKUS is close to the body surface, and since there are few flat parts, scanning tends to be unstable. One advantage is that the contour of the bone becomes a landmark, but there is the drawback that the part covered by the bone cannot be evaluated. In addition, a more specialized evaluation method such as dynamic ultrasound is required.

The diversity and specificity of the site of such musculoskeletal injuries make it more difficult to acquire MSKUS skills, and hinder the development of US equipment specialized for musculoskeletal evaluation.

That said, do not just give up. It is necessary to devise optimal imaging methods and imaging conditions one by one beginning with the clinically important sites in musculoskeletal injuries, and make steady efforts to standardize them. For these, automation of imaging, automatic discrimination function, etc. can be effectively utilized.

Currently, MSKUS for sports injuries is mainly performed by doctors, but its use is expected to increase more and more in the fields of rehabilitation and sports medicine. To this end, it is necessary for academic societies to play a central role in creating appropriate imaging guidelines for all sports injuries and making efforts to disseminate correct imaging methods.

Publisher's Note Springer Nature remains neutral with regard to jurisdictional claims in published maps and institutional affiliations. 